\title{
Design and Research of Virtual Experiment based on Virtual Reality
}

\author{
Jia Liu, Yu Fan
}

Haikou College of Economics, Haikou, Hainan, 571100

Keywords: Virtual Experiment, Virtual Reality, System Construction

\begin{abstract}
As an important part of modern distance education, virtual experiment plays an irreplaceable role in guaranteeing teaching effect, and has gained more and more people's favor. But because of its late development, technically very immature. The current virtual experiment is a simple two-dimensional experimental demonstration experiment, in the experimental realism, interactivity and teaching effects and other aspects of the real experiment is very different.
\end{abstract}

\section{Introduction}

Virtual experiment is a branch of modern distance education, through the use of a variety of computer multimedia and network communication technology to simulate the function of various experimental instruments to make up for the lack of distance teaching conditions, so that students can not rely on computer software to simulate hardware Do all kinds of experiments, get the same experience with the real experiment, so enrich the perceptual knowledge, deepen the understanding of teaching content. The virtual experiment into the experimental class, on the one hand to solve the experimental equipment and equipment, high cost of funding; on the other hand, coordination of the laboratory less, less equipment, students use more problems; also enable students to experiment from time and region Restrictions, and other changes in the experimental equipment and function changes only in the software can be changed, a great savings in cost. So the virtual experiment has very important research significance.

\section{Virtual Reality Technology}

Virtual reality technology is a new field of computer research, in recent years gradually attention for all sectors, and in the application areas have been further developed. It is a collection of high-tech, bringing together computer graphics, computing simulation technology, multimedia technology, artificial intelligence technology, simulation technology, man-machine interface technology, sensor technology, graphics image real-time generation technology, network technology and human behavior Research and many other key technologies. The high level of integration and penetration of these technologies enables virtual reality technology to give users a realistic environment experience that allows users to devote themselves to this environment by using a variety of special devices to explore the macroscopic world and the microscopic world for a variety of reasons The movement of things to observe the law of change. The definition of virtual reality technology can be summarized as follows: Virtual reality technology is a realistic simulation of human in the natural environment of visual, auditory and sports behavior of human-computer interface technology. It uses 3D visualization system, multi-sensing interactive technology and high-resolution display technology of scientific visualization technology to generate three-dimensional realistic virtual environment, the user with a specific device, you can enter the virtual space, a virtual environment, a real-time Interact, perceive and manipulate the various objects in the virtual world, thus gaining immersive feelings and experiences.

Virtual reality must be generated by the computer three-dimensional space, that is, "virtual environment", the user through the visual, auditory, power and other perceived environment, in addition to viewing, but also can operate some of the objects, and the space of the object to communicate. And in the space with the user's freedom to move and control the scene changes, and thus produce a considerable sense of integration and participation, as if immersive in general. Real, 
interactive, plot and other characteristics of the virtual reality is unique charm. Virtual reality technology is essentially an advanced computer user interface technology. So far the computer man-machine interface is machine-oriented, terminal, keyboard, mouse, printer and other interface devices, are suitable for computer-specific equipment. Virtual reality in the man-machine interface, it is user-oriented. In a virtual reality system, users interact with the virtual environment in a natural way. The so-called natural way, refers to the user through the visual, auditory, tactile and other sensory virtual environment, so that users in the real environment of the illusion. At the same time, the user through the real environment in the behavior, to intervene in the virtual environment. Therefore, the virtual reality of the human interface device, completely different from the existing computer man-machine interface device.

\section{Virtual Reality System Construction}

According to the specific application of virtual reality technology to develop different virtual reality system, virtual reality system consists of four parts:

1) virtual scene, that is, an interactive virtual environment, can be viewed and observed from any angle, it is a three-dimensional model or environment defined by the database, is achieved through the software;

2) computer with virtual reality software, the system to provide real-time observation and participation in the virtual world's ability;

3) I / O interface device, providing man-machine interface for users to achieve interaction. Including input devices and output devices. Input devices are used to observe and construct virtual worlds such as: data gloves, floating mice, torque balls, etc .; output devices are used to display images of virtual worlds, such as: helmet displays. CRT terminal and so on.

4) the user, the operator of the virtual scene.

The I / O interface device provides a user-to-computer interface, which is generated by dedicated software combined with database technology. When the user receives the task, through the I / O interface device to access the computer, then the computer will extract the virtual scene, through the virtual reality software to explain, and then provided to the user, which is the user can access the virtual scene. Using the input and output devices, the user can interact with the scene to cause real-time changes in the scene, as in the real way of interaction.

Immersive virtual system is a more complex system. The immersive sense created by the virtual reality system relies heavily on the sensory input (including visual, auditory, tactile, etc.) provided by various hardware, which makes the participant fully into a virtual realm and can not see the real world. Users must wear a helmet, hand gloves and other sensor tracking device in order to interact with the virtual world. Because this system can be the user's vision, hearing and external isolation, so the user's surrounding real environment is effectively shielded, so that participants have a virtual realm surrounded by the feeling, can be fully into the virtual world go with. The advantage of this system is that users can completely immerse themselves in the virtual world, the disadvantage is that the system equipment is expensive, difficult to popularize the promotion.

Augmented reality technology allows participants to see objects in the real world while superimposing the graphics of the virtual environment on real objects. The penetrating head-mounted display overlays the computer-generated graphics with the actual real-time environment of the participant. For example, after a maintenance engineer wears a penetrating head-mounted display, the printer can be repaired by observing a pattern that overlaps with a laser printer. These graphics may provide various graphical arrows, illustrations and instructions that can even guide his maintenance. Enhance the reality of the system depends on the virtual reality location tracking technology to achieve accurate overlap, but the current technology can not do this completely.

The desktop virtual reality system is a virtual reality system which realizes the virtual instrument and the simulation simulation of the experiment process through the computer programming technology. It only uses personal computers and workstations to carry out the simulation, the computer screen as a participant to observe the virtual realm of the window, the user through the 
keyboard, the mouse can interact with the virtual environment. Users in the ordinary computer through the computer screen can be observed within 360 degrees of virtual realm, truly feel the virtual scene. With the mouse and the keyboard, the object can be manipulated, but the participant lacks full immersion because the participant sits in front of the monitor and observes the virtual realm. But it is simple structure, low cost, easy to popularize, is a set of economical and practical system. Common desktop virtual reality technologies are: virtual reality-based virtual reality Quick VR, virtual reality modeling language VRML and so on.

\section{Realization Scheme of Virtual Experiment System Based on Virtual Reality}

The use of virtual reality technology to build a virtual experimental system is a system of work, must do a good job planning, step by step. Can be divided into the following steps: 1) to analyze the task to master the shape of each virtual instrument features, physical characteristics, functional characteristics. To detail the dimensions, position, and interface modeling of its various parts, draw a tree hierarchy diagram for modeling purposes. Get the instrument's texture data, which is captured primarily by a digital camera. The use of the various instruments and equipment used in the experiment, including the use of cables and other interfaces, parts and the number of statistics in order to reuse. Clear the process of experimentation, the activities of each step action, clear the contents of human-computer interaction. 2) Create a model Create a virtual instrument model through modeling tools, including geometric modeling and physical modeling. Uniform output for ".wrl" format. 3) Optimize the model to optimize the model to create a good, reduce the unnecessary nodes, remove the redundant polygon to reduce the model size. 4) tune the texture of the virtual instrument to add texture effects, for different shapes of objects using different texture mapping, the texture mapping to the corresponding surface of the object. 5) beautify the modification to the object by adding light, color and material to make it look more realistic. The final modification of the built model. 6) Add the interaction According to the design of the experimental process will be divided into different scenes. In each experiment scenario, the interactive function is added to the built model according to the action of each step, so that it can meet the requirements of the virtual experiment. 7) interface design experimental body part built, you must have a friendly interface. Design the user interface according to the experimental process for user convenience. 8) Integrated publishing Virtual experiments must be published on the Internet to be used for distance education. So the final integration of virtual experiments on the page, the Internet release. In order to facilitate the use of students, should provide the use of help and related plug-in download.

In order to make the user truly immersed in the virtual experiment environment, the virtual experiment instrument must be made realistic and natural, so the virtual instrument modeling is the foundation of the whole virtual experiment system. People's perception of information is mainly through visual, auditory, tactile, olfactory and gravity to get, a good virtual scene must provide these senses with the display environment similar to the stimulus. $80 \%$ of human information is obtained through the eyes, so the quality of visual perception in the user's subjective perception of the environment occupies the most important position. So the virtual instrument 3D visual modeling work is the primary consideration. Virtual reality 3D visual modeling has its own characteristics compared with other graphic modeling systems, mainly in the following three aspects:

1) virtual reality can have a very wide range of objects, often need to build a large number of completely different types of objects.

2) Virtual reality, some objects must have their own behavior, other graphics modeling system is often just to build static objects, even if there is movement, it is often a relatively simple form of fixed.

3) Objects in virtual reality must be able to respond to observers. When an observer interacts with an object, the object must react in a proper manner.

Physical modeling is a higher level of modeling in virtual reality systems. The physical modeling of the virtual instrument includes the lighting, material, mass, weight, hardness, shape change pattern of the object, which combines with the behavior rules of geometric modeling to form a more 
realistic virtual instrument model. It needs physics and computer graphics with, involving mechanical problems, mainly weight modeling, surface deformation and softness and other physical properties of the embodiment. Physical Modeling In order to make the VR reliable and locate the user in it, the virtual object must be as true. At least the solids should not pass through each other, and the objects should be moved in the intended way when being pushed and pulled. The physical properties of VR are often described by differential equations, which constitute the dynamics system. This kinetic system is studied by system analysis and system simulation. System simulation is actually a physical simulation of the dynamics system.

\section{Conclusion}

In this paper, the development of computer network virtual experiment system as an example, research and discussion of the system to achieve the key and difficult, and ultimately through the realization of the various parts of them organically together to form a high performance virtual reality-based computer Network virtual experiment system, so that the virtual reality based on the virtual experimental system development methods and implementation techniques to practice and test. The system is based on Web development, can be used for practical teaching, has a strong practical value. The construction method and implementation technology adopted in the system development process can be applied to the development of general virtual experiment system.

\section{References}

[1] Liu Xiaolan, Zhang Wei. The type and development trend of virtual laboratory [J] .Application Research of Computers. 2004 (11)

[2] Qu Zhao. Online virtual experiment teaching [J]. Chinese University Teaching. 2004 (07)

[3] Ouyang Xingming, Yao Xiaolong, Xie Xinrong. Network Virtual Laboratory Design and Implementation [J]. Computer Engineering. 2004 (02)

[4] Zhou Xianxiang, Jiang Shizhong, Jiang Guohua. Virtual reality technology research status and progress [J]. Computer simulation. 2003 (07)

[5] Wang Ruchuan, Yao Xumin, Wang Haiyan, Liu Li.Study on Virtual Traffic Communication Based on Java and VRML [J]. Journal of System Simulation, 2003 (07)

[6] Yang Yuhang, Li Huijie, Feng Yuncheng. Electronic Sandbox Simulation System Based on Virtual Reality Technology [J]. Computer Simulation. 2003 (01)

[7] Jia Zhenyuan, Song Li, Guo Lisa. Virtual instrument development status and characteristics [J]. Instrument Technology. 2002 (05) 